\title{
The inflammasome potentiates influenza/Staphylococcus aureus superinfection in mice
}

\author{
Keven M. Robinson, ${ }^{1}$ Krishnaveni Ramanan, ${ }^{1}$ Michelle E. Clay, ${ }^{2}$ Kevin J. McHugh, ${ }^{3}$ \\ Matthew J. Pilewski, ${ }^{1}$ Kara L. Nickolich, ${ }^{1}$ Catherine Corey, ${ }^{4,5}$ Sruti Shiva, ${ }^{4,5}$ Jieru Wang, ${ }^{3}$ \\ Radhika Muzumdar, ${ }^{3}$ and John F. Alcorn ${ }^{3}$ \\ 'Department of Medicine, University of Pittsburgh School of Medicine, Pittsburgh, Pennsylvania, USA. \\ ${ }^{2}$ Department of Microbiology and Immunology, Geisel School of Medicine at Dartmouth, Hanover, New Hampshire, USA. \\ ${ }^{3}$ Department of Pediatrics, Children's Hospital of Pittsburgh of UPMC, Pittsburgh, Pennsylvania, USA. \\ ${ }^{4}$ Department of Pharmacology and Chemical Biology, and ${ }^{5}$ Vascular Medical Institute, University of Pittsburgh School of \\ Medicine, Pittsburgh, Pennsylvania, USA.
}

Secondary bacterial respiratory infections are commonly associated with both acute and chronic lung injury. Influenza complicated by bacterial pneumonia is an effective model to study host defense during pulmonary superinfection due to its clinical relevance. Multiprotein inflammasomes are responsible for IL-1 $\beta$ production in response to infection and drive tissue inflammation. In this study, we examined the role of the inflammasome during viral/bacterial superinfection. We demonstrate that $\mathrm{ASC}^{-/-}$mice are protected from bacterial superinfection and produce sufficient quantities of IL-1 $1 \beta$ through an apoptosis-associated speck-like protein containing CARD (ASC) inflammasome-independent mechanism. Despite the production of IL-1 $\beta$ by $A_{S C^{-1-}}$ mice in response to bacterial superinfection, these mice display decreased lung inflammation. A neutrophil elastase inhibitor blocked ASC inflammasome-independent production of IL-1 $\beta$ and the IL-1 receptor antagonist, anakinra, confirmed that IL-1 remains crucial to the clearance of bacteria during superinfection. Delayed inhibition of NLRP3 during influenza infection by MCC950 decreases bacterial burden during superinfection and leads to decreased inflammatory cytokine production. Collectively, our results demonstrate that ASC augments the clearance of bacteria, but can also contribute to inflammation and mortality. ASC should be considered as a therapeutic target to decrease morbidity and mortality during bacterial superinfection.

Conflict of interest: The authors have declared that no conflict of interest exists.

Submitted: September 13, 2017 Accepted: February 28, 2018 Published: April 5, 2018

Reference information: JCI Insight. 2018;3(7):e97470. https:// doi.org/10.1172/jici.insight.97470

\section{Introduction}

Viral respiratory tract infections are common in both children and adults and can cause significant morbidity and mortality. Viruses can disrupt physical barriers and alter immunologic responses leading to increased susceptibility to secondary bacterial infections. Clinicians face difficult diagnostic and management challenges when confronted with a symptomatic patient who has an upper respiratory infection. It can be difficult to determine if symptoms are caused by a viral infection or a viral/bacterial superinfection. Children with upper respiratory viruses commonly develop concurrent acute otitis media, sinusitis, or pneumonia (1). A classic example of viral/bacterial superinfection is influenza complicated by secondary bacterial pneumonia. Mouse models using influenza and Staphylococcus aureus can be used as a tool to study viral-bacterial synergy and immune mechanisms of pathogenesis.

Influenza A is a highly transmittable respiratory virus that causes seasonal epidemics and intermittent pandemics. Severe pathogenesis during influenza infection is commonly a result of secondary bacterial pneumonia and leads to increased hospitalizations, health care utilization, and mortality (2-4). Currently, $S$. aureus is the most predominant bacterial organism that causes bacterial pneumonia following influenza infection. Both methicillin-sensitive $S$. aureus (MSSA) and methicillin-resistant $S$. aureus (MRSA) commonly complicate influenza (5). Increased understanding of the immune response and alteration of host defense that occurs during influenza and predisposes to secondary bacterial pneumonia is crucial to decreasing mortality associated with this disease. 
IL-1 $\beta$ is a proinflammatory cytokine that is produced following activation of pattern recognition receptors by microbial products, which initiates IL-1 $\beta$ gene expression and synthesis of pro-IL- $1 \beta$. IL- $1 \beta$ drives neutrophil and macrophage recruitment, activates the release of other cytokines important to host defense, TNF- $\alpha$ and IL-6, and drives $\mathrm{T}_{\mathrm{H}} 17$ differentiation of naive $\mathrm{T}$ cells (6). Inflammasomes are multiprotein complexes that are assembled after sensing pathogen-associated molecular patterns (PAMPs) derived from pathogens and recruit inactive pro-caspase-1. Oligomerization of pro-caspase-1 allows for generation of active caspase-1, which is a protease that cleaves pro-IL-1 $\beta$ into mature and active IL-1 $\beta$ (7). Multiple inflammasome complexes have been discovered consisting of NOD-like receptors (NLRs) or absent in melanoma 2-like (AIM2-like) receptors, adaptor protein apoptosis-associated speck-like protein containing CARD (ASC), and pro-caspase-1. Upon stimulation, NLRs or AIM2 oligomerize to a caspase-1-containing scaffold and allow for cleavage of pro-IL-1 $\beta$ or pro-IL-18 into mature IL-1 $\beta$ or IL-18. In addition, inflammasome-independent mechanisms exist for processing IL-1 $\beta$. Serine proteases such as neutrophil elastase, proteinase-3 (PR3), and cathepsin G can process pro-IL-1 $\beta$ into mature IL-1 $\beta$ that helps modulate host defense against invading pathogens $(8,9)$.

We have previously shown that mice infected with both influenza A and $S$. aureus have increased bacterial burden and higher mortality compared with mice infected with singular $S$. aureus infection (10). We have demonstrated that preceding influenza attenuates $S$. aureus-induced IL-1 $\beta$ production and subsequent type 17 immunity, increasing susceptibility to secondary bacterial pneumonia (11). Our current work explores the role of different components of multiprotein-complex inflammasomes that coordinate to transform pro-IL-1 $\beta$ into mature IL-1 $\beta$. We show that ASC augments the clearance of bacteria during influenza infection and also contributes to excessive inflammation and increased mortality. In addition, we demonstrate that $A S C^{-1-}$ mice can produce IL-1 $\beta$ through inflammasome-independent mechanisms and that ASC is a potential therapeutic target to decrease mortality during influenza/bacterial superinfection.

\section{Results}

ASC inflammasome contributes to decreased bacterial clearance, increased mortality, and lung inflammation during influenza and bacterial superinfection. To determine the role of inflammasome components during influenza complicated by secondary bacterial pneumonia, we challenged wild-type (WT) C57BL/6 mice with $100 \mathrm{PFU}$ of influenza A/PR/8/34 H1N1 or control PBS for 6 days followed by $1 \times 10^{8} \mathrm{CFU}$ MSSA or control PBS for 24 hours. We found that Pycard (ASC) expression is upregulated in both singular influenza infection and mice superinfected with influenza and MSSA (Figure 1A) compared with naive WT mice. In addition, Pycard expression is increased in superinfected mice compared with mice that received MSSA alone. Aim2 is upregulated during both singular MSSA infection and singular influenza infection compared with naive WT mice (Figure 1B), although when directly comparing MSSA to influenza/MSSA, there is decreased mRNA expression in the superinfected mice. Nlrp3 expression is upregulated during superinfection compared with naive WT mice (Figure $1 \mathrm{C}$ ). To further evaluate the specific role of ASC, we infected both WT and $A S C^{-1-}$ mice with A/PR/8/34 followed by MSSA challenge. We saw enhanced bacterial clearance (approximately $55 \%$ decreased bacterial burden) in the superinfected $A S C^{-/-}$mice compared with superinfected WT mice (mean CFU WT Flu/ SA 2,352 \pm 376 ; mean CFU $A S C^{-/}$Flu/SA 1,055 \pm 191) (Figure 1D). Consistent with prior studies, we saw increased bacterial burden in the superinfected WT mice compared with WT mice singularly infected with MSSA. Despite differences in bacterial burden between the groups, there was no difference in viral burden between the WT and $A S C^{-1-}$ mice in either superinfection or mice singularly infected with influenza (Figure 1E). Additionally, when challenged with a lethal dose of MSSA during influenza infection, we observed increased survival in $A S C^{-1-}$ mice compared with WT mice (Figure $1 F)$. These findings reveal a critical role for the ASC inflammasome during influenza and MSSA superinfection, as it contributes to the inhibition of bacterial clearance. Up to $75 \%$ of pediatric influenzaassociated deaths are found to have positive cultures for $S$. aureus at a normally sterile site, either MSSA or MRSA. For this reason, we also infected WT and $A S C^{-1-}$ mice with influenza A/PR/8/34 followed by MRSA challenge. We saw enhanced bacterial clearance (approximately $60 \%$ decreased bacterial burden) in the superinfected $A S C^{-1}$ mice compared with superinfected WT mice (mean CFU WT Flu/MRSA 46,383 \pm 9,511; mean CFU $A S C^{-1}$ Flu/SA 18,118 $\pm 6,176$ ) (Figure 1G). These data support the notion that the ASC protein could play an important role in postviral pneumonia caused by different strains of $S$. aureus. 
A

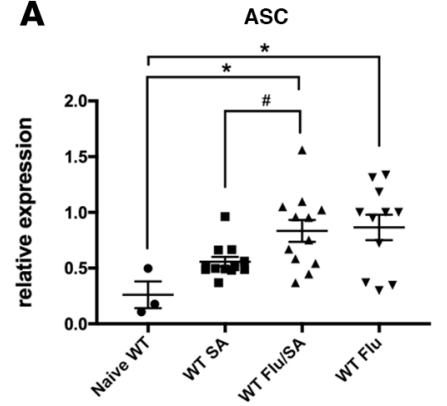

B

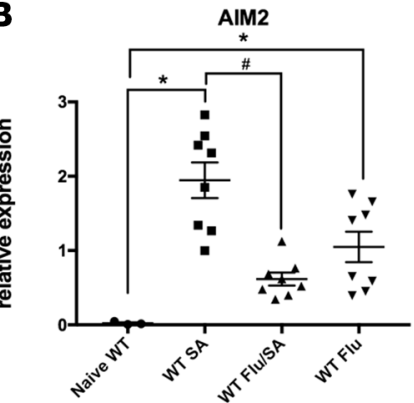

C

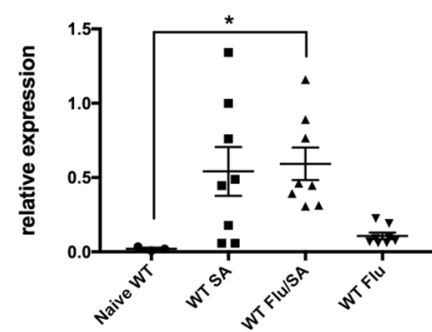

D

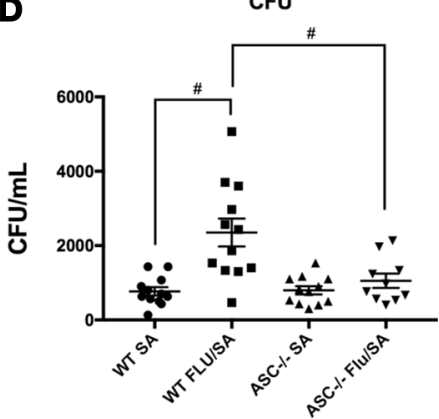

E

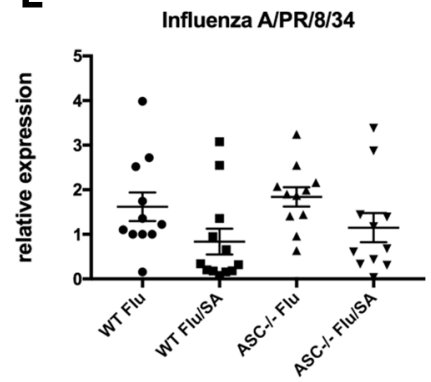

$\mathbf{F}$

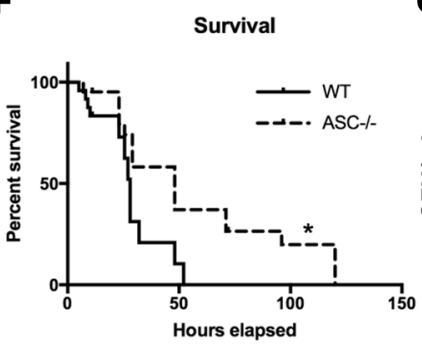

G CFU

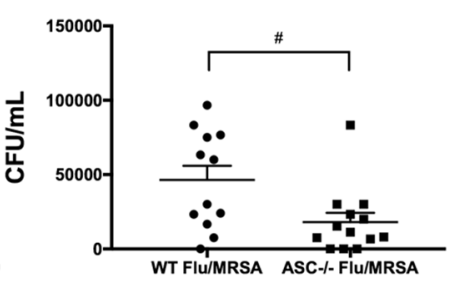

Figure 1. The ASC inflammasome promotes decreased bacterial clearance and increased mortality during influenza and bacterial superinfection. $C 57 \mathrm{BL} / 6$ mice were infected with 100 PFU of influenza A/PR/8/34 or vehicle for 6 days, and then challenged with $10^{8}$ CFU of methicillin-sensitive Staphylococcus aureus (MSSA) or vehicle for 24 hours. (A-C) Gene expression of inflammasome components in lung $(n=3-12)$. C57BL/6 and $A S C^{-1-}$ mice were infected with $100 \mathrm{PFU}$ of influenza A/PR/8/34 or vehicle for 6 days, and then challenged with $10^{8} \mathrm{CFU}$ of MSSA or vehicle for 24 hours. (D) Bacterial colony counts in lung homogenate $(n=10-12)$. (E) Gene expression of influenza matrix protein in lung $(n=11-12)$. (F) Survival curve for WT and $A S C^{-1-}$ mice challenged with influenza followed by MSSA $(n=8)$. (C) C57BL/ 6 mice were infected with $100 \mathrm{PFU}$ of influenza A/PR/8/34 or vehicle for 6 days, and then challenged with $5 \times 10^{7}$ CFU of methicillin-resistant S. aureus (MRSA) or vehicle for 24 hours $(n=12) .{ }^{*} P<0.05$ versus naive WT by 1 -way ANOVA; ${ }^{\#} P<0.05$ comparing SA with Flu/ $S A$ by unpaired $t$ test. Data points reflect individual values \pm SEM. Each experiment was independently performed at least twice and data are shown from combined experiments with the exception of naive mouse data, which was performed once.

We hypothesized that the protection seen in $\mathrm{ASC}^{-/-}$mice during influenza and bacterial superinfection was related to a decreased hyperinflammatory response. To evaluate this, we collected bronchoalveolar lavage fluid (BALF) and measured neutrophil and macrophage recruitment to the lung. We found that superinfected $A S C^{-1-}$ mice had decreased numbers of both neutrophils (approximately $67 \%$ decrease) and macrophages (approximately 50\% decrease) compared with WT mice (Figure 2A). As seen previously, there were higher numbers of neutrophils and macrophages in superinfected WT mice compared with those that received MSSA alone. Additionally, we measured levels of both inflammatory and antiinflammatory cytokines/chemokines in lung homogenate and found decreased levels of IL-6 and G-CSF (approximately 40\% decrease) in superinfected $A S C^{-/-}$compared with WT mice. IL-10 was also decreased in the superinfected $A S C^{-/}$mice compared with WT (approximately $60 \%$ decrease), although there was no difference in TNF- $\alpha$ (Figure 2, B-E). Overall, the $A S C^{-1-}$ mice demonstrated decreased inflammatory cytokine responses compared with the WT controls. We next assessed the oxygen consumption rate $(\mathrm{OCR})$ of $\mathrm{CD} 11 \mathrm{c}^{+}$cells from WT and $A S C^{-1}$ superinfected mice. OCR was measured at the basal level, and then sequentially treated with oligomycin, carbonyl cyanide 4-(trifluoromethoxy)phenylhydrazone (FCCP), and antimycin-A plus rotenone. Both basal OCR and the maximal OCR reached after FCCP injection were similar in WT and $A S C^{-/-}$cells (Figure $2 \mathrm{~F}$ ), suggesting that differences in mitochondrial respiration of lung antigen-presenting cells is not the reason for enhanced bacterial clearance in the $A S C^{-1-}$ mice during superinfection. Furthermore, we have previously shown that preceding influenza inhibits type 17 immunity and IL-17- and IL-22-associated antimicrobial peptides that are necessary for bacterial clearance in the lung. We measured gene expression of IL-17- and IL-22-associated antimicrobial peptides and found that there was no difference between WT and $A_{S C}{ }^{-1}$ superinfected mice (Supplemental Figure 1; supplemental material available online with this article; 
A

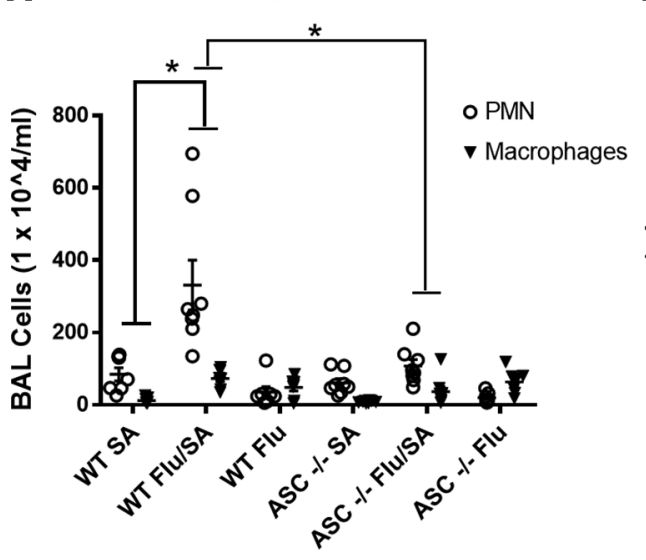

B

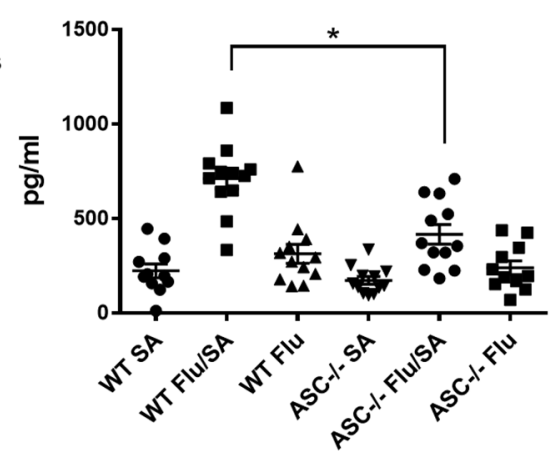

C

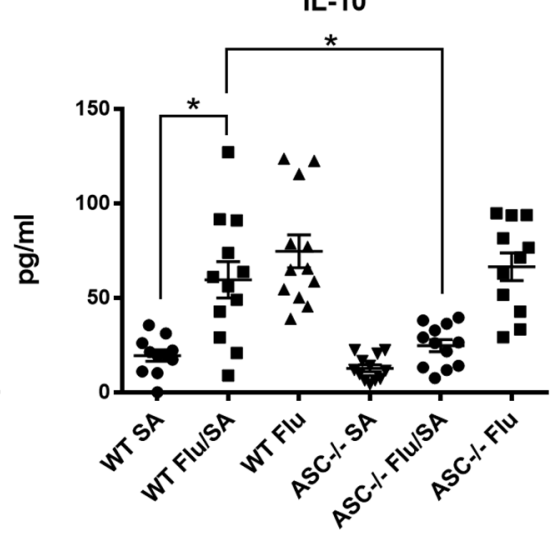

D

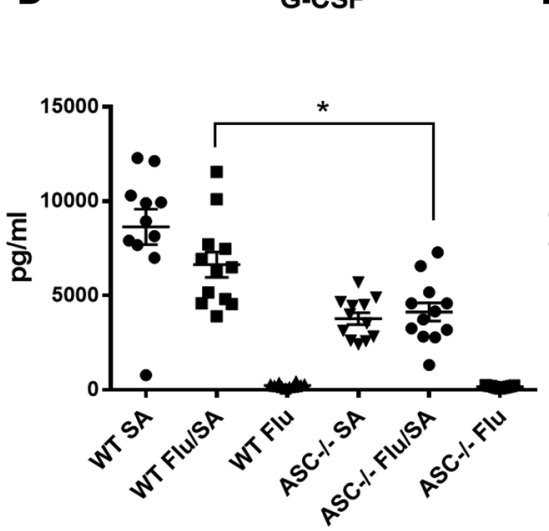

E

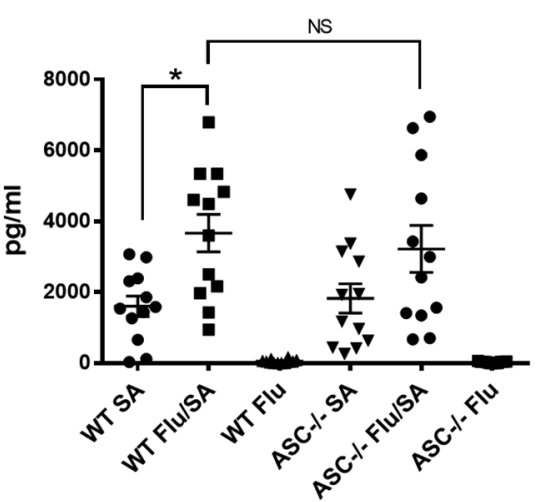

F

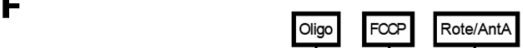

Figure 2. The ASC inflammasome drives increased inflammation during influenza and bacterial superinfection. $C 57 B L / 6$ and $A S C^{-/-}$mice were infected with 100 PFU of influenza A/PR/8/34 or vehicle for 6 days, and then challenged with $10^{8}$ CFU of methicillin-sensitive Staphylococcus aureus (MSSA) or vehicle for 24 hours. (A) Bronchoalveolar lavage (BAL) cell differential counts $(n=7-8)$. (B-E) Cytokine concentrations in lung homogenate as measured by Bio-Plex immunoassay $(n=11-12)$. ${ }^{*} P<0.05$ versus Flu/MSSA by unpaired $t$ test. Data points reflect individual values \pm SEM. Each experiment was independently performed at least twice and data are shown from combined experiments. (F) Oxygen consumption rate (OCR) of CD11 ${ }^{+}$cells isolated from WT and $A S C^{-/-}$measured at the basal level and then after sequential treatment with $1 \mu \mathrm{M}$ oligomycin, $1.5 \mu \mathrm{M}$ FCCP, and $0.1 \mu \mathrm{M}$ rotenone plus $1 \mu \mathrm{M}$ antimycin-A using the XFe-96 extracellular flux assay system. The assay is representative of 3 independent experiments, run in triplicate, and the mean values \pm SEM are shown. NS, not significant.

https://doi.org/10.1172/jci.insight.97470DS1), suggesting that accentuation of type 17 immunity is not the reason for the improved bacterial clearance of MSSA in $\mathrm{ASC}^{-/-}$superinfected mice.

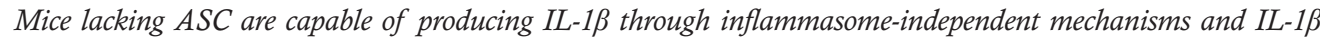
remains crucial to the effective clearance of bacteria during superinfection. ASC protein is present in both NLR and non-NLR inflammasomes and $\mathrm{ASC}^{-/}$mice represent a tool to broadly investigate how inflammasome deletion affects immunity and host defense. Upon stimulation by pathogens, NLRs or AIM2 oligomerize with caspase-1-containing scaffolds and allow for cleavage of pro-IL-1 $\beta$ into mature IL-1. IL-1 $\beta$ recruits neutrophils and macrophages, and activates the release of proinflammatory cytokines. To determine if the decreased inflammatory response seen in $A S C^{-1}$ superinfected mice is a result of decreased IL-1 $\beta$, we measured protein levels in both BALF and lung homogenate. Levels of IL-1 $\beta$ are decreased in the BALF of superinfected $A S C^{-1-}$ mice compared with WT (approximately 27\% decrease) (Figure 3A), although there is no difference in the levels of IL-1 $\beta$ in lung homogenate (Figure $3 \mathrm{~B}$ ). Although naive $A S C^{-1-}$ mice have decreased levels of IL-1 $\beta$ in lung homogenate compared with WT mice (Supplemental Figure 2), $A S C^{-1-}$ mice are able to produce IL-1 $\beta$ in response to both singular MSSA infection and influenza (MSSA superinfection). Since there are inflammasome-independent mechanisms for the secretion of active IL-1 $\beta$ related to serine proteases such as neutrophil elastase, PR3, and cathepsin G, we treated both WT and $A S C^{-1-}$ mice with sivelestat, a neutrophil elastase inhibitor, during superinfection. We found the WT and $A S C^{-1-}$ mice that received the neutrophil elastase inhibitor cleared bacteria equally effectively (Figure 3C). Furthermore, 
A

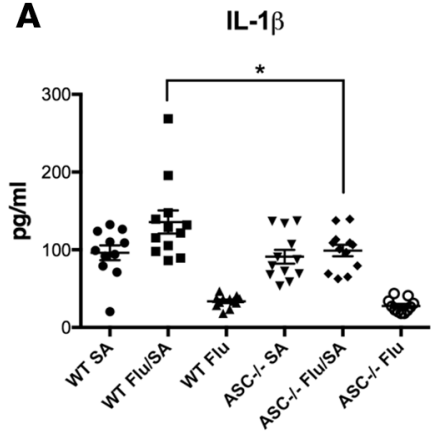

E

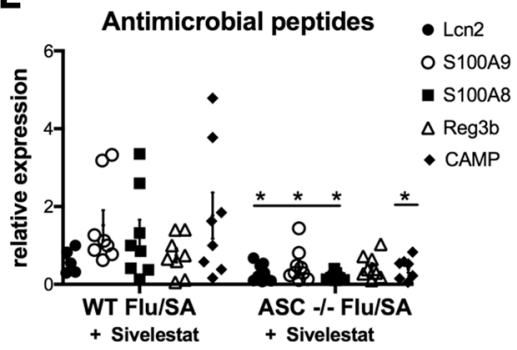

B

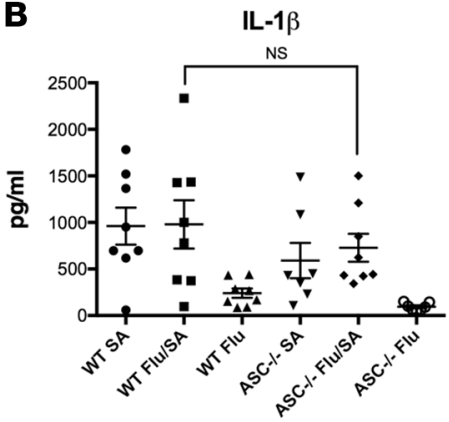

C

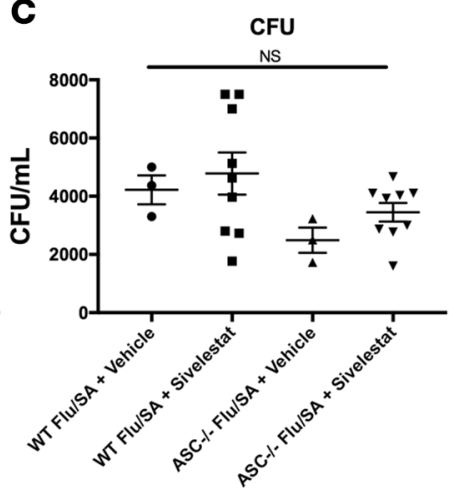

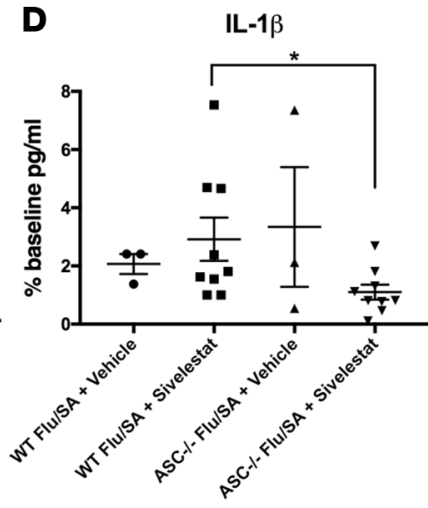

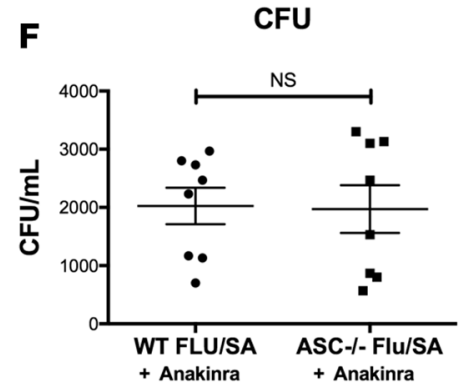

Figure 3. Mice lacking the ASC inflammasome produce IL-1 $\beta$ through inflammasome-independent mechanisms and IL-1 $\beta$ is crucial to the effective clearance of bacteria during superinfection. $C 57 \mathrm{BL} / 6$ and $A S C^{-1-}$ mice were infected with influenza $A / P R / 8 / 34$ or vehicle for 6 days, and then challenged with $10^{8} \mathrm{CFU}$ of methicillin-sensitive Staphylococcus aureus (MSSA) or vehicle for 24 hours. (A) IL-1 $\beta$ concentrations in bronchoalveolar lavage fluid as measured by Bio-Plex immunoassay $(n=11-12)$. (B) IL-1 $\beta$ concentrations in lung homogenate as measured by ELISA $(n=7-8)$. C57BL/6 and $A S C^{-1-}$ mice were infected with influenza for 6 days, and then challenged with methicillin-resistant S. aureus (MRSA) for 24 hours. Mice received silvestat, $10 \mu \mathrm{g} / \mathrm{g}$ i.p. or vehicle control, on days 4-6 after influenza. (C) Bacterial colony counts in lung homogenate $(n=9)$. (D) IL-1 $\beta$ concentrations in lung homogenate as measured by Bio-Plex immunoassay $(n=6)$. (E) Gene expression of type 17 immunity-related antimicrobial peptides $(n=7-8)$. C57BL/6 and $A S C^{-1-}$ mice were infected with influenza for 6 days, and then challenged with MSSA for 24 hours. Mice received anakinra, $600 \mu \mathrm{g}$ in $200 \mu$ l of PBS i.p., on days 0-6 after influenza. (F) Bacterial colony counts in lung homogenate $(n=8) .{ }^{*} P<0.05$ versus Flu/SA by 1-way ANOVA or unpaired $t$ test. Data points reflect individual values \pm SEM. Each experiment was independently performed at least twice and data are shown from combined experiments. NS, not significant.

there were decreased levels of IL-1 $\beta$ in the $A S C^{-/}$mice that received neutrophil elastase inhibitor compared with WT controls (mean $2.9 \%$ baseline WT Flu/SA/sivelestat mice compared with mean $1.1 \%$ baseline $\mathrm{ASC}^{-/-} \mathrm{Flu} / \mathrm{SA} /$ sivelestat) (Figure 3D). These findings reveal that inflammasome-independent IL-1 $\beta$ production plays a critical role during influenza/S. aureus superinfection. Because IL-1 $\beta$ is known to induce polarization of IL-17-producing cells and subsequently affect the expression of IL-17- and IL-22-producing antimicrobial peptides, we measured gene expression of Reg3 $\alpha \beta \gamma, L c n 2, S 100 A 8, S 100 A 9$, and CAMP in these mice and found decreased levels of $L c n 2, S 100 A 8, S 100 A 9$, and CAMP mRNA in the ASC-1 mice that received the neutrophil elastase inhibitor compared with WT controls (Figure $3 \mathrm{E}$ ). The reduction of IL-17 and IL-22-related antimicrobial peptides is likely due to a reduction in IL-1 $\beta$ in the sivelestat-treated $A S C^{-1}$ mice, from deletion of the inflammasome combined with the neutrophil elastase inhibitor, and is the reason why bacteria are not cleared as well. This is not seen in sivelestat-treated WT mice due to functional inflammasome-mediated IL-1 $\beta$ production. We have previously reported that IL-1 $\beta$ critically regulates IL-17- and IL-22-related antimicrobial peptides during influenza and secondary bacterial pneumonia (12).

During influenza/S. aureus superinfection, active IL-1 $\beta$ is produced by both inflammasome-dependent and inflammasome-independent mechanisms. To confirm that IL-1 $\beta$ plays a role during superinfection, we treated both WT and $A S C^{-/}$mice with anakinra, an IL-1 receptor (IL-1R) antagonist, and found that similar to mice treated with neutrophil elastase inhibitor, there was no longer a difference in bacterial clearance between the $A S C^{-1-}$ mice and WT controls (Figure 3F). These data indicate that in the absence of active IL-1 $\beta, A S C^{-/-}$mice are no longer protected during influenza/S. aureus superinfection.

Next, we examined caspase-1, the enzyme that activates when assembled into the inflammasome complex. We measured gene expression of caspase- 1 and found no differences in mRNA levels (Figure 4A). We measure protein levels of the proenzyme form of caspase- 1 and the activated p20 subunit and unexpectedly found 
A

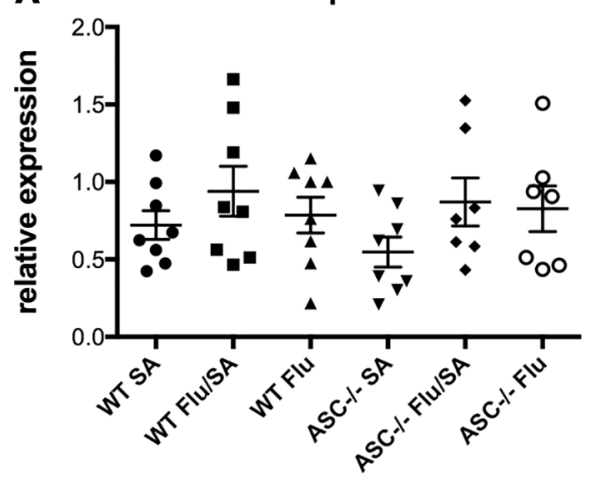

D

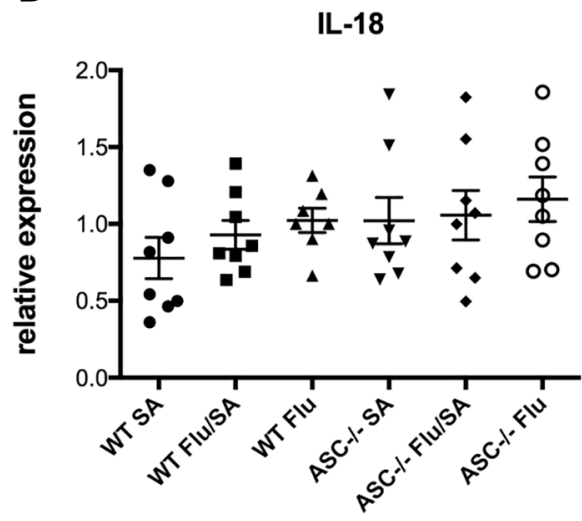

B

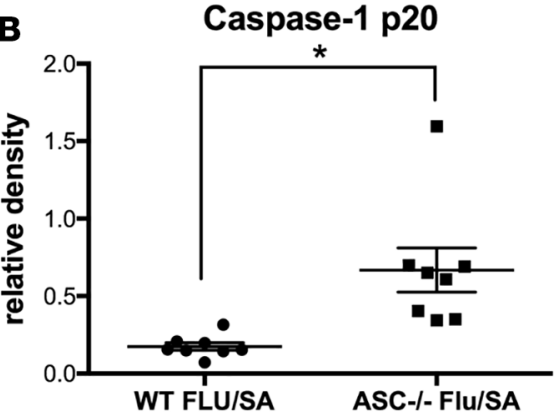

E

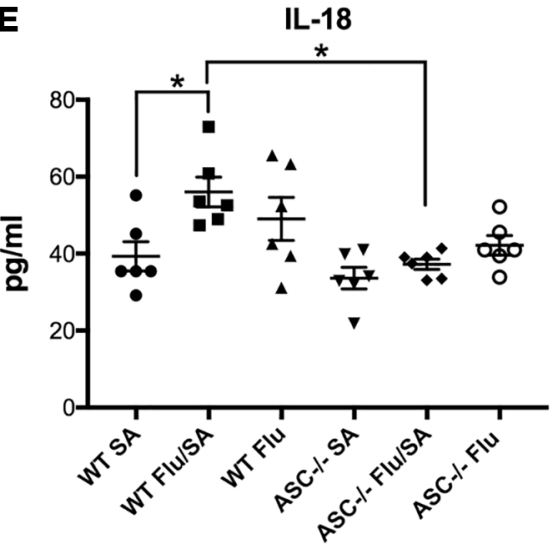

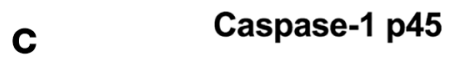

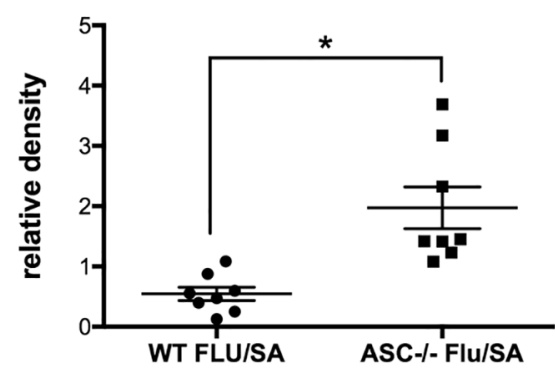

$\mathbf{F}$

Figure 4. IL-18 does not alter bacterial clearance during superinfection. C57BL/6 and $A S C^{-1-}$ mice were infected with influenza $A / P R / 8 / 34$ or vehicle for 6 days, and then challenged with $10^{8}$ CFU of methicillin-sensitive Staphylococcus aureus (MSSA) or vehicle for 24 hours. (A) Gene expression of caspase-1 ( $n=$ 7-8). (B and C) Protein measurement of caspase-1 p20 and caspase-1 p45 products measured by Western blot $(n=8)$. (D) Gene expression of IL-18 $(n=7-8)$. (E) IL-18 concentrations measured by ELISA $(n=6)$. C57BL/6 mice were infected with influenza A/PR/8/34 for 6 days, received $200 \mu \mathrm{g}$ of IL-18 inhibitor or IgG control, and then challenged with $10^{8} \mathrm{CFU}$ of MSSA 4 hours later and harvested at 24 hours. (F) CFU in lung homogenate $(n=6)$. ${ }^{*} P<0.05$ versus Flu/ SA by 1-way ANOVA or unpaired $t$ test. Data points reflect individual values \pm SEM. Each experiment was independently performed at least twice and data are shown from combined experiments with the exception of the IL-18 inhibition experiment that was performed once. NS, not significant.

increased levels of both forms of caspase-1 in the $A S C^{-1}$ superinfected mice compared with WT superinfected mice (Figure 4, B and C, and Supplemental Figures 3 and 4). Similar to IL-1 $\beta$, the inflammasome regulates production of the proinflammatory cytokine IL-18 by activating intracellular caspase-1, which cleaves proIL-18 into active IL-18. To determine if $A S C^{-/}$superinfected mice have decreased levels of IL-18, we measured gene expression and protein levels of IL-18. We found no differences in gene expression of IL-18 (Figure 4C) and decreased levels of IL-18 (approximately 34\% decrease) in lung homogenate of superinfected $\mathrm{ASC}^{-/}$mice compared with WT (Figure 4D). We hypothesized that decreased IL-18 in the $A S C^{-1-}$ mice may be associated with increased bacterial killing and more effective bacterial clearance. Prior literature has shown that $I 118^{-1}$ mice have decreased septicemia as a result of $S$. aureus bacteremia compared with WT controls (13). To test this hypothesis, we treated WT mice with an IL-18 inhibitor during influenza/MSSA superinfection, and found that there was no difference in bacterial clearance between mice that received the IL-18 inhibitor and mice that received an isotype control (Figure 4E). We confirmed that the IL-18 inhibitor had been effective by measuring gene expression of IL-36, which IL-18 is known to induce (14) (Supplemental Figure 5). Therefore, although $\mathrm{IL}-18$ is decreased in $A S C^{/-}$mice during superinfection, it is unlikely to be the reason these mice clear bacteria more effectively during superinfection.

The non-NLR inflammasome AIM2 contributes to poor bacterial clearance, while temporal NLR inhibition enhances bacterial clearance during influenza A/S. aureus superinfection. Multiple inflammasome complexes have been discovered consisting of different sensor molecules, such as NLRs or AIM2-like receptors. The ASC protein is present in both NLR and non-NLR inflammasomes and while $A S C^{-1}$ mice represent a tool to broadly investigate the inflammasome, we used other methods to interrogate different components of the inflammasome 
A

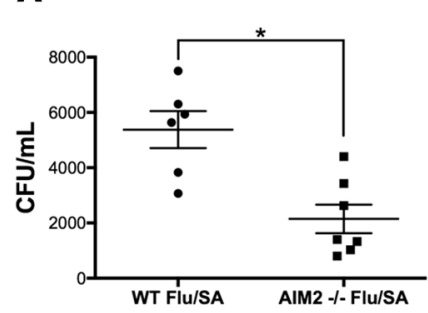

B

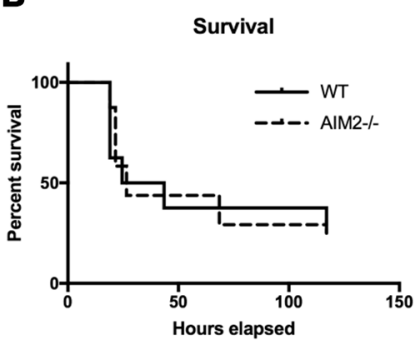

c

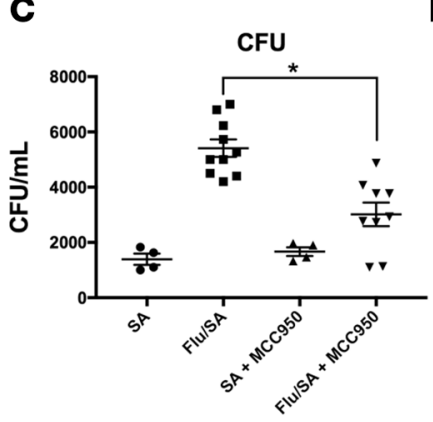

D

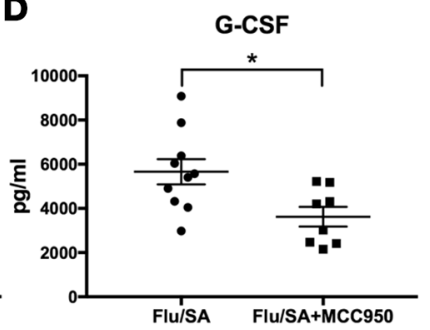

E

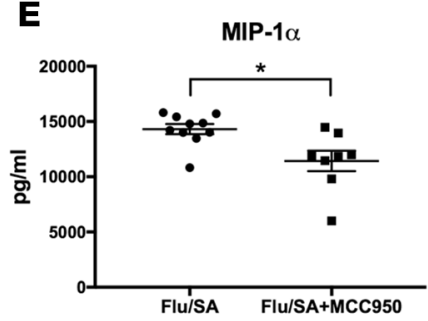

$\mathbf{F}$

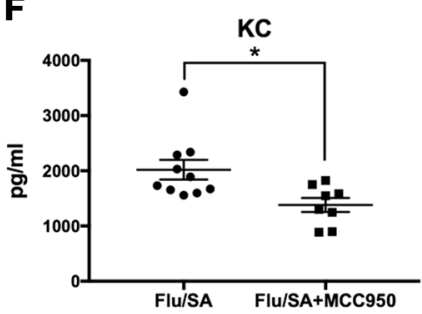

G

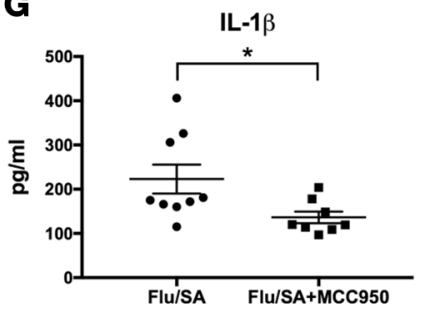

H

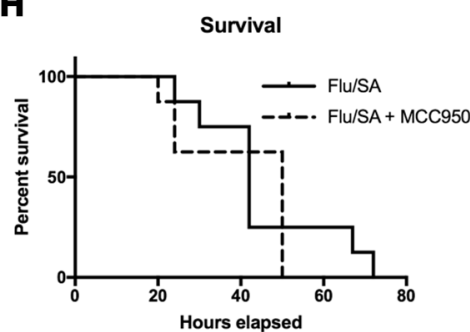

Figure 5. Temporal NLRP3 inhibition enhances bacterial clearance but does not affect mortality during influenza and bacterial superinfection. C57BL/6 and $A / M^{-1-}$ mice were infected with influenza $A / P R / 8 / 34$ for 6 days, and then challenged with $10^{8} \mathrm{CFU}$ of methicillin-sensitive Staphylococcus aureus (MSSA) for 24 hours. (A) Bacterial colony counts in lung homogenate $(n=6)$. (B) Survival curve for WT and $A^{\prime} M^{-/-}$mice challenged with influenza followed by MSSA $(n=7-8)$. C57BL/ 6 mice were infected with $100 \mathrm{PFU}$ of influenza A/PR/8/34 or vehicle for 6 days, and then challenged with $10^{8}$ CFU of MSSA or vehicle for 24 hours. Mice received MCC950 $5 \mathrm{mg} / \mathrm{kg}$ by oropharyngeal aspiration or PBS control on days 4 and 6 after influenza. (C) Bacterial colony counts in lung homogenate ( $n=4$ in MSSA-alone groups and 9-10 in Flu/MSSA groups). (D-F) Cytokine concentrations in lung homogenate as measured by BioPlex immunoassay $(n=9-10)$. (C) IL-1 $\beta$ concentrations in lung homogenate as measured by ELISA $(n=9-10)$. (H) Survival curve for WT and MCC950-treated mice challenged with influenza followed by MSSA $(n=8)$. ${ }^{*} P<0.05$ versus Flu/MSSA by 1 -way ANOVA or unpaired $t$ test. Data points reflect individual values \pm SEM. Each experiment was independently performed at least twice and data are shown from combined experiments with the exception of $A^{\prime} M^{-1-}$ mouse data displayed in panel $\mathbf{A}$ and survival curves, which were performed once.

during influenza and bacterial superinfection. As previously discussed, transcription of the inflammasome sensor molecules Aim2 and Ntrp3 is upregulated during either singular MSSA infection or dual influenza/ MSSA infection (Figure 1, B and C). Studies have shown that both NLRP3 and AIM2 are required for inflammation during influenza infection through the use of mice lacking components of NLRP3 and AIM2 (15, 16). However, temporal inhibition of NLRP3 during influenza infection through the use of a small-molecule inhibitor of NLRP3 results in decreased disease and lung inflammation if used later in the course of infection $(17,18)$. To investigate the individual component AIM2, we challenged WT and $A I M 2^{-/-}$mice with A/ $\mathrm{PR} / 8 / 34$ for 6 days followed by MSSA and saw enhanced bacterial clearance in the superinfected $A I M 2^{-1-}$ mice compared with superinfected WT mice (Figure 5A). However, there was no difference in weight loss, which can serve as a marker of morbidity, between the 2 groups (data not shown). When challenged with a lethal dose of MSSA during influenza infection, we observed similar survival in $A I M 2^{-/-}$mice compared with WT mice (Figure 5B). Then, to investigate the individual component NLRP3, we challenged WT mice with A/PR/8/34 for 6 days followed by MSSA. Mice were treated with MCC950 or control on days 1, 3, and 5 after influenza. MCC950 treatment throughout the course of influenza infection resulted in increased bacterial burden at 24 hours after MSSA challenge (Supplemental Figure 6), consistent with increased mortality during single influenza infection (17). Next, mice received A/PR/8/34 or PBS control for 6 days followed by MSSA and were treated with MCC950 or control on days 4 and 6 after influenza. Delayed MCC 950 treatment resulted in decreased bacterial burden at 24 hours after MSSA challenge compared with control (Figure 5C). In addition, G-CSF, keratinocyte chemoattractant (KC), and macrophage inflammatory protein $1 \alpha(\mathrm{MIP}-1 \alpha)$ levels in the lung homogenate were significantly reduced in the MCC950-treated mice (Figure 5, D-F). We also observed a decrease (approximately 40\% change) in IL-1 between the 2 groups (Figure $5 \mathrm{G}$ ). Despite differences in bacterial burden, there was no difference in weight loss (data not shown) or when challenged with a lethal dose of MSSA, survival, between the 2 groups (Figure $5 \mathrm{H}$ ). These data suggest that deletion of AIM2 or inhibition of NLRP3 can enhance bacterial clearance but do not affect morbidity or mortality. 


\section{Discussion}

In this report, we assess the in vivo contribution of the inflammasome and IL-1 $\beta$ to host immune responses during influenza complicated by secondary bacterial pneumonia. We use both genetically engineered mice and protein inhibitors to distinguish that IL-1 $\beta$ is important for effective bacterial clearance during viral/ bacterial superinfection. Furthermore, we establish a role for different components of the inflammasome. ASC contributes to decreased bacterial clearance, excessive inflammation, and increased mortality. These observations have important clinical implications for the prevention of bacterial superinfection in the lung during influenza infection and may be applicable to secondary bacterial infections in the context of other primary lung injuries including both acute and chronic stimuli.

Bacterial superinfection of the lung during influenza infection promotes severe disease pathogenesis and leads to increased mortality. In recent years, increased efforts have focused on establishing how preceding influenza infection leads to an ineffective immune response to secondary bacterial pneumonia. We have previously shown that preceding influenza limits the host response by leading to decreased production of IL-1 $\beta$, IL-17, and IL-22 in response to secondary superinfection with $S$. aureus $(10,12)$. The restoration of IL-1 $\beta$ during the course of influenza rescued the induction of IL-17 and IL-22 by $S$. aureus and improved bacterial clearance (12). Although IL-1 $\beta$ promotes the polarization of $\mathrm{CD}^{+}$cells to $\mathrm{T}_{\mathrm{H}} 17$ cells that produce IL-17 and IL-22, cytokines that are necessary for the effective clearance of $S$. aureus, IL-1 $\beta$ also promotes inflammation through multiple mechanisms including, but not limited to, cytokine production and neutrophil recruitment. Excessive inflammation can lead to lung injury and potentially death.

Previous reports have investigated the role of both IL-1 $\beta$ and NLRP3 in $S$. aureus pneumonia and found that mice lacking the IL-1R are just as or more susceptible to $S$. aureus, depending on the strain used, and have increased bacterial burden compared with WT controls $(12,19)$. Mice lacking NLRP3 had equivalent bacterial burden but decreased inflammation compared with WT controls (19), potentially suggesting that bacterial clearance and survival are related to IL- $1 \beta$ signaling and NLRP3 activation is related to the development of lung injury and inflammation. Others have investigated $S$. aureus in a skin infection model and demonstrated that mice deficient in IL-1 $\beta$ and ASC are both more susceptible to infection; however in this study, $A S C^{-1-}$ mice produced less IL-1 $\beta$ compared with WT controls (20). Importantly, $A S C^{-1-}$ mice used in our studies were capable of producing sufficient amounts of IL- $1 \beta$. Although this contrasts with other studies utilizing $A S C^{-1}$ mice, there are previously published reports that $A S C^{-1-}$ mice can produce IL-1 $\beta$ in response to Pseudomonas aeruginosa pneumonia through an inflammasome-independent mechanism (21). The $S$. aureus toxin leukocidin A/B has been shown to have differential requirements for both ASC and NLRP3 depending on the cellular contact site, i.e., intra- or extracellular (22), indicating that the strain of $S$. aureus and its associated virulence factors and the location of infection may lead to different requirements of components of the inflammasome.

Prior work has also examined the role of IL-1 $\beta$, NLRP3, and ASC during influenza infection. Influenza A H1N1 is known to induce IL-1 $\beta$ (23) and mice deficient in IL-1R1 have decreased lung inflammation early in the course of infection (day 3) but increased inflammation later in the course of infection (day 9) and decreased survival $(24,25)$. NLRP3 and ASC are both upregulated by influenza by day 3 after infection but are decreased by day 7 after infection (15). In addition, Nlrp $3^{-/-}$mice have been shown to be both protected and susceptible to influenza, depending on the study, while $A S C^{-1-}$ mice have previously been shown to be susceptible to influenza infection $(15,16,26)$. The variability in susceptibility to influenza infection in $A S C^{-1-}$ mice represents discord between previously published work and the data presented here and could be related to differences in virus preparation. In addition, it has been established that dedicator of cytokinesis 2 (DOCK2) expression in bone marrow-derived DCs and bone marrow-derived macrophages differs between different $A S C^{-/-}$mouse strains, and Ippagunta et al. have proposed that differences in genetic background and/or environmental factors could have caused this divergent expression of DOCK2, which regulates the migration of lymphocytes (27). Of note, DOCK2 production is present in our $A S C^{-1-}$ strain, as confirmed by Western blot (data not shown). This, or other unidentified differences, could explain the varied results seen in $\mathrm{ASC}^{-1}$ strains in response to influenza infection. More recently, a small-molecule inhibitor specific for NLRP3, MCC950, has been used to reassess the role of NLRP3 during influenza infection. Studies have demonstrated that temporal inhibition of NLRP3, later in the course of influenza infection, protects mice from severe influenza infection $(17,18)$. This finding was intriguing because of the timing of the clinical development of secondary bacterial pneumonia during influenza infection, which typically occurs 5-7 days after influenza infection. 
We have previously established that IL-1 $\beta$ is important for bacterial clearance during influenza complicated by bacterial superinfection. However, given the proinflammatory nature of IL-1 $\beta$, we also had concerns that restoration of IL-1 $\beta$ in humans could lead to devastating consequences. Higher levels of IL-1 $\beta$ have been associated with poor outcomes in acute respiratory distress syndrome $(28,29)$. Prior animal studies using the IL-1R antagonist, anakinra, have demonstrated that IL-1 signaling controls $S$. aureus burden in the lung and the use of anakinra may be harmful during pneumonia (30). In this report, we confirm that IL-1 $\beta$ is important for the effective clearance of $S$. aureus during influenza infection, but our results also suggest that the inflammasome component ASC can lead to excessive inflammation and increased mortality during influenza/S. aureus superinfection. IL-1 $\beta$ will likely be a difficult therapeutic immune target during influenza infection to establish a balance between decreasing inflammation, but aid in effective clearance if a secondary bacterial pneumonia develops in the lung. Based on our findings, there would be great potential to study ASC as a therapeutic target if a small-molecule inhibitor could be developed to inhibit ASC interactions with caspase-1.

Our work demonstrates that mice lacking ASC can produce robust quantities of IL-1 $\beta$ comparable to WT mice during viral and bacterial superinfection. This is noteworthy because investigators will have to take into account all the mechanisms by which IL-1 $\beta$ is produced during infection as additional therapeutics are developed that interrogate the IL- $1 \beta$ axis.

In this report, we used a small-molecule specific inhibitor of NLRP3, MCC950, to interrogate temporal NLRP3 inhibition during influenza/MSSA superinfection. When given during the entire course of influenza infection, similar to genetic deletion, NLRP3 inhibition was detrimental and led to increased bacterial burden in the lung. Prior studies have shown that IL-1 $\beta$ is markedly reduced in Nlrp $3^{-/-}$mice during influenza infection (15), and these data support our conclusions that some IL-1 $\beta$ is necessary for bacterial clearance of $S$. aureus during viral infection. In contrast to the experiments in which NLRP3 was inhibited during the entire course of infection, total ASC deletion led to improved bacterial clearance. The most likely reason for the difference in these results is that ASC-knockout mice are still producing robust amounts of IL-1 $\beta$ during superinfection. When given later during influenza infection, NLRP3 inhibition resulted in decreased bacterial burden in the lung. As expected, there was decreased IL-1 $\beta$ in the mice that received the NLRP3 inhibitor, but there was still a significant amount of IL-1 $\beta$ present. In addition, NLRP3 inhibition resulted in decreased proinflammatory cytokines G-CSF, MIP-1 $\alpha$, and KC. Although we observed differences in bacterial burden and proinflammatory cytokines during delayed NLRP3 inhibition, there were no differences in weight loss or mortality. Delayed NLRP3 inhibition decreases mortality during singular influenza infection (17), but did not confer survival protection during secondary bacterial superinfection in our model. It is difficult to interpret how this would translate into a clinical patient scenario, and because of the significant improvement in mortality during singular influenza infection and lack of harm during viral/bacterial superinfection, NLRP3 inhibition should still be considered as a novel therapeutic target during influenza to reduce severity of infection. Additional studies are needed to examine NLRP3 inhibition in the context of other bacterial superinfections.

Our study provides the groundwork for experimentation regarding ASC as a therapeutic target during influenza and secondary bacterial pneumonia. The data provide increased understanding of how IL- $1 \beta$ is produced during bacterial superinfection, by both inflammasome-dependent and inflammasome-independent mechanisms. Future studies will be needed to determine the therapeutic potential of ASC deletion or inhibition during acute and chronic respiratory infections.

\section{Methods}

Animal studies. Six- to eight-week-old male WT, C57BL/6 mice were purchased from Taconic Farms. $A S C^{-1-}$ mice were provided by Genentech through Uma Nagarajan (University of North Carolina, Chapel Hill, North Carolina, USA). $A I M^{--}$mice were provided by Jeiru Wang and Radhika Muzumdar (University of Pittsburgh). Mice were maintained under pathogen-free conditions at the Children's Hospital of Pittsburgh of UPMC. All of the studies used age- and sex-matched mice. In specific experiments mice were treated as indicated with neutrophil elastase inhibitor (sivelestat, $10 \mu \mathrm{g} / \mathrm{g}$ in $200 \mu \mathrm{l}$ PBS i.p.; Santa Cruz Biotechnology, catalog sc203398) or PBS vehicle control; recombinant human IL-1R antagonist (anakinra, Swedish Orphan Biovitrum, $600 \mu \mathrm{g}$ in $200 \mu \mathrm{l}$ of PBS i.p.); IL-18 inhibitor (monoclonal antibody anti-mouse IL-18 (BioXCell, catalog BE0327; $200 \mu \mathrm{g}$ in $200 \mu 1$ of PBS i.p.) or PBS vehicle control; or a selective inhibitor of NLRP3 (MCC950, Selleckchem, catalog S7809; 5 mg/ $\mathrm{kg}$ in $50 \mu \mathrm{PBS}$ by oropharyngeal aspiration) or PBS vehicle control. 
Bacterial and viral infections. MSSA (ATCC, 49775) or MRSA (USA 300) (provided as a gift by Alice Prince, Columbia University, New York, New York, USA) was cultured as detailed by ATCC instructions in casein hydrolysate yeast extract-containing modified medium for 18 hours to stationary growth phase. Mice were inoculated with $1 \times 10^{8} \mathrm{CFU}$ of MSSA or $5 \times 10^{7} \mathrm{CFU}$ of MRSA in $50 \mu$ sterile PBS by oropharyngeal aspiration. Influenza A/PR/8/34 H1N1 was used to inoculate mice with $100 \mathrm{PFU}$ of influenza (in $50 \mu 1$ sterile PBS) by oropharyngeal aspiration. Viral burden was determined by quantitative real-time RT-PCR on lung RNA for viral matrix protein as previously described (31). Mice were sequentially challenged with influenza or vehicle (PBS) for 6 days followed by infection with bacteria or vehicle (PBS) for an additional 24 hours.

Analysis of lung inflammation. At the indicated time points, mouse lungs were lavaged with $1 \mathrm{ml}$ sterile PBS for inflammatory cell differential counts. The cranial lobe of the right lung was homogenized in sterile PBS by mechanical grinding. The resulting lung homogenate was used for bacterial colony counting and cytokine analysis by Bio-Plex Multiplex immunoassay (Bio-Rad) or by ELISA for IL-1 $\beta$ (R\&D Systems). Middle and caudal lobes of the right lung were snap-frozen and homogenized under liquid nitrogen for RNA extraction by an RNA isolation kit (Agilent Technologies). RNA analysis was performed by standard RT-PCR using Assay-on-Demand TaqMan probes and primers (Applied Biosystems).

Cell isolation. Lungs from infected mice were cut into small pieces $(\sim 2 \mathrm{~mm})$ and digested with collagenase. Digested lungs then were forced through a $70-\mu \mathrm{m}$ filter, and RBC were lysed. CD $11 \mathrm{c}^{+}$and $\mathrm{CD}_{11 \mathrm{c}^{-}}$cells then were isolated using Miltenyi positive selection microbeads per the manufacturer's instructions (Miltenyi Biotec).

Measurement of OCR. The OCR of $\mathrm{CD}_{11} \mathrm{c}^{+}$cells isolated from WT and $A S C^{-1-}$ mice was measured at the basal level. Cells were treated with $1 \mu \mathrm{M}$ oligomycin, $1.5 \mu \mathrm{M}$ FCCP, and $0.1 \mu \mathrm{M}$ rotenone plus $1 \mu \mathrm{M}$ antimycin-A using the XFe-96 extracellular flux assay system (Seahorse Biosciences). Cells were plated in triplicate. Seahorse assays were performed in unbuffered Dulbecco's Modified Eagle Medium (DMEM) supplemented with $2.5 \mu \mathrm{M}$ glucose, $1 \mu \mathrm{M}$ pyruvate, and $2 \mu \mathrm{M}$ glutamine.

Statistics. All of the data are presented as the mean \pm SEM. Significance was tested by the unpaired $t$ test (for 2 means) or 1-way ANOVA (for multiple data groups), followed by Tukey's post hoc test. All studies were repeated 2 or 3 times and collected data were combined for the figures unless otherwise described. Significance of survival data was assessed by the Gehan-Brislow-Wilcoxon test. Data were analyzed using GraphPad Prism software. $P<0.05$ was considered significant.

Study approval. Mouse experiments were conducted with approval from the University of Pittsburgh Institutional Animal Care and Use Committee.

\section{Author contributions}

KMR designed the research studies, conducted experiments, acquired data, analyzed data, and drafted the manuscript. KR, MEC, KJM, MJP, and KLN conducted experiments and acquired data. CC and SS provided reagents and equipment, and analyzed data. JW provided reagents and mouse strains. RM provided mouse strains. JFA designed the research studies, provided reagents, analyzed data, and drafted the manuscript.

\section{Acknowledgments}

This work was supported by a Parker B. Francis Fellowship (to K.M. Robinson), NIH NHLBI K08 HL133445 01 (to K.M. Robinson), and NIH NHLBI R01 HL107380 (to J.F. Alcorn).

Address correspondence to: John F. Alcorn, Division of Pulmonary Medicine, Allergy, and Immunology, Department of Pediatrics, Children's Hospital of Pittsburgh of UPMC, One Children's Hospital Drive, 9127 Rangos, 4401 Penn Avenue, Pittsburgh, Pennsylvania 15224, USA. Phone: 412.692.7595; Email: john.alcorn@chp.edu.

1. Hendaus MA, Jomha FA, Alhammadi AH. Virus-induced secondary bacterial infection: a concise review. Ther Clin Risk Manag. 2015;11:1265-1271.

2. Randolph AG, et al. Critically ill children during the 2009-2010 influenza pandemic in the United States. Pediatrics. 2011;128(6):e1450-e1458.

3. Williams DJ, et al. Influenza coinfection and outcomes in children with complicated pneumonia. Arch Pediatr Adolesc Med. 2011;165(6):506-512.

4. Morens DM, Taubenberger JK, Fauci AS. Predominant role of bacterial pneumonia as a cause of death in pandemic influenza: implications for pandemic influenza preparedness. J Infect Dis. 2008;198(7):962-970. 
5. Wong KK, et al. Influenza-associated pediatric deaths in the United States, 2004-2012. Pediatrics. 2013;132(5):796-804.

6. Dinarello CA. Immunological and inflammatory functions of the interleukin-1 family. Annu Rev Immunol. 2009;27:519-550.

7. Broz P, Dixit VM. Inflammasomes: mechanism of assembly, regulation and signalling. Nat Rev Immunol. 2016;16(7):407-420.

8. Netea MG, Simon A, van de Veerdonk F, Kullberg BJ, Van der Meer JW, Joosten LA. IL-1beta processing in host defense: beyond the inflammasomes. PLoS Pathog. 2010;6(2):e1000661.

9. Guo H, Callaway JB, Ting JP. Inflammasomes: mechanism of action, role in disease, and therapeutics. Nat Med. 2015;21(7):677-687.

10. Kudva A, et al. Influenza A inhibits Th17-mediated host defense against bacterial pneumonia in mice. J Immunol. 2011;186(3):1666-1674.

11. Robinson KM, et al. Influenza A virus exacerbates Staphylococcus aureus pneumonia in mice by attenuating antimicrobial peptide production. J Infect Dis. 2014;209(6):865-875.

12. Robinson KM, et al. Influenza A exacerbates Staphylococcus aureus pneumonia by attenuating IL-1 $\beta$ production in mice. J Immunol. 2013;191(10):5153-5159.

13. Wei XQ, et al. Altered immune responses and susceptibility to Leishmania major and Staphylococcus aureus infection in IL18-deficient mice. J Immunol. 1999;163(5):2821-2828.

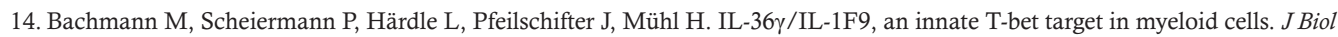
Chem. 2012;287(50):41684-41696.

15. Allen IC, et al. The NLRP3 inflammasome mediates in vivo innate immunity to influenza A virus through recognition of viral RNA. Immunity. 2009;30(4):556-565.

16. Zhang H, et al. AIM2 inflammasome is critical for influenza-induced lung injury and mortality. J Immunol. 2017;198(11):4383-4393

17. Tate MD, et al. Reassessing the role of the NLRP3 inflammasome during pathogenic influenza A virus infection via temporal inhibition. Sci Rep. 2016;6:27912.

18. Coates BM, et al. Inhibition of the NOD-like receptor protein 3 inflammasome is protective in juvenile influenza A virus infection. Front Immunol. 2017;8:782.

19. Kebaier C, et al. Staphylococcus aureus $\alpha$-hemolysin mediates virulence in a murine model of severe pneumonia through activation of the NLRP3 inflammasome. J Infect Dis. 2012;205(5):807-817.

20. Miller LS, et al. Inflammasome-mediated production of IL-1beta is required for neutrophil recruitment against Staphylococcus aureus in vivo. J Immunol. 2007;179(10):6933-6942.

21. Patankar YR, Mabaera R, Berwin B. Differential ASC requirements reveal a key role for neutrophils and a noncanonical IL-1 $\beta$ response to Pseudomonas aeruginosa. Am J Physiol Lung Cell Mol Physiol. 2015;309(8):L902-L913.

22. Melehani JH, Duncan JA. Inflammasome activation can mediate tissue-specific pathogenesis or protection in Staphylococcus aureus infection. Curr Top Microbiol Immunol. 2016;397:257-282.

23. Kim KS, Jung H, Shin IK, Choi BR, Kim DH. Induction of interleukin-1 beta (IL-1 $\beta$ ) is a critical component of lung inflammation during influenza A (H1N1) virus infection. J Med Virol. 2015;87(7):1104-1112.

24. Schmitz N, Kurrer M, Bachmann MF, Kopf M. Interleukin-1 is responsible for acute lung immunopathology but increases survival of respiratory influenza virus infection. J Virol. 2005;79(10):6441-6448.

25. Guo L, et al. Pulmonary immune cells and inflammatory cytokine dysregulation are associated with mortality of IL-1R1-/mice infected with influenza virus (H1N1). Zool Res. 2017;38(3):146-154.

26. Ichinohe T, Lee HK, Ogura Y, Flavell R, Iwasaki A. Inflammasome recognition of influenza virus is essential for adaptive immune responses. J Exp Med. 2009;206(1):79-87.

27. Ippagunta SK, et al. Addendum: defective Dock2 expression in a subset of ASC-deficient mouse lines. Nat Immunol. 2012;13(7):701-702.

28. Meduri GU, et al. Persistent elevation of inflammatory cytokines predicts a poor outcome in ARDS. Plasma IL-1 beta and IL-6 levels are consistent and efficient predictors of outcome over time. Chest. 1995;107(4):1062-1073.

29. Luo XR, Wu WL, Wang L, Ye XQ, Zhang D. [Changes in interleukin-1beta in serum and bronchoalveolar lavage fluid in the patient with acute respiratory distress syndrome]. Zhongguo Wei Zhong Bing Ji Jiu Yi Xue. 2003;15(6):365-366.

30. Labrousse D, et al. Kineret/IL-1ra blocks the IL-1/IL-8 inflammatory cascade during recombinant Panton Valentine Leukocidin-triggered pneumonia but not during S. aureus infection. PLoS One. 2014;9(6):e97546.

31. Van der Velden J, Janssen-Heininger YM, Mandalapu S, Scheller EV, Kolls JK, Alcorn JF. Differential requirement for c-Jun N-terminal kinase 1 in lung inflammation and host defense. PLoS One. 2012;7(4):e34638. 